\title{
Association of the s1799724 and rs1800629 Polymorphisms of the TNF- $\alpha$ Gene with Susceptibility to Cervical Cancer, a Systematic Review and Meta-Analysis Based on 24 Case-Control Studies
}

\author{
Sedigheh Hamadani ${ }^{1,2}$, Mahdieh Kamali ${ }^{1,2}$, Sedigheh Hantoushzadeh ${ }^{1,2}$, Razieh \\ Sadat Tabatabaee $^{3}$, Hossein Neamatzadeh ${ }^{4}$, EInaz Foroughi ${ }^{5}$, Fatemeh Haghighi ${ }^{3}$, \\ Masoud Zare-Shehneh ${ }^{6}$, Elham Alsadat Hejazian-Yazdi ${ }^{6}$
}

${ }^{1}$ Department of Perinatology, School of Medicine, Tehran University Medical of Sciences, Tehran, Iran. ${ }^{2}$ Maternal-Fetal and Neonatal Research Center, Tehran University of Medical Sciences, Tehran, Iran. ${ }^{3}$ Department of Gynecology and Obstetrics, Shahid Sadoughi University of Medical Sciences, Yazd, Iran. ${ }^{4}$ Mother and Newborn Health Research Center, Shahid Sadoughi University of Medical Sciences, Yazd, Iran. ${ }^{5}$ Department of Pediatric Dentistry, Arak university of Medical Sciences, Arak, Iran. ${ }^{6}$ Department of Medical Genetics, International Campus, Shahid Sadoughi University of Medical Sciences, Yazd, Iran. ${ }^{6}$ Department of Physical Education, Yazd Branch, Islamic Azad University, Yazd, Iran.

\begin{abstract}
Objective: Some studies have recently focused on the association between TNF- $\alpha$ polymorphisms and cervical cancer; however, results have been inconsistent. In order to drive a more precise estimation, the present systematic review and meta-analysis is performed to investigate the relationship of the TNF- $\alpha$ rs 1800629 and s1799724 polymorphisms with cervical cancer risk. Methods: An electronic search was conducted on PubMed, Web of Science, and Google scholar databases, for papers that describe the association between TNF- $\alpha$ polymorphisms and cervical cancer risk. Results: A number of 24 case-control studies in 22 publications were identified according to the inclusion criteria. The results showed that rs1800629 polymorphism was significantly associated with the increased cervical cancer risk under four genetic models (A vs. G: OR $=1.277,95 \%$ CI: 1.104-1.477, p $=0.001$; AA vs. GG: OR = 1.333, 95\% CI: 1.062-1.674, p=0.013; AG vs. GG: OR = 1.307, 95\% CI: $1.064-$ $1.605, \mathrm{p}=0.011$; and $\mathrm{AA}+\mathrm{AG}$ vs. $\mathrm{GG}: \mathrm{OR}=1.324,95 \% \mathrm{CI}: 1.104-1.587, \mathrm{p}=0.002)$. In stratified analysis, there was a significant association between rs 1800629 polymorphism and cervical cancer risk in the subgroup of Caucasians and African, but not in Asians. However, no statistically significant association was observed between the s1799724 and cervical cancer risk under all genetic models. Furthermore, stratification by ethnicity indicated no association between the s1799724 and cervical cancer risk. Conclusion: the present meta-analysis suggests that the rs 1800629 polymorphism of the TNF- $\alpha$ gene was significantly associated with cervical cancer risk, but not s1799724.
\end{abstract}

Keywords: TNF- $\alpha$ gene- cervical cancer- polymorphism- meta-analysis

\section{Introduction}

Cervical cancer is the third most common female cancer (after breast and colon cancer) worldwide [1-2], and the number-one cause of cancer-related death in women in developing countries [3-4]. Cervical cancer is an important preventable cause of morbidity and mortality among women worldwide [5]. It is divided into two types: Cervical squamous cell carcinoma, which is derived from squamous cells and cervical adenocarcinoma, arising in

Corresponding Author:

Dr. Mahdieh Kamali

Department of Perinatology, School of Medicine, Tehran University Medical of Sciences, Tehran, Iran.

Maternal-Fetal and Neonatal Research Center, Tehran University of Medical Sciences, Tehran, Iran.

Email: mahdiehkamali.md@gmail.com 
the glandular cells of cervix. Cervical cancer incidence varies widely in different countries, ranging from around 3 to over 40 per 100,000 [6]. The higher incidence rates are seen mainly in developing countries, particularly in Africa, and the lowest rates occur in Western Europe and west Asia.

The major risk factor for cervical cancer is infection with human papillomavirus (HPV) [5]. However, several epidemiological studies have pointed out the importance of genetic risk factors in cervical cancer. Evidence revealed that familial clustering of cervical cancer and its precursor forms [7]. In 1999, Magnusson et al. reported a significant familial aggregation for cervical cancer [8]. Genes of the immune response system have been studied to investigate the potential association with cervical cancer as well as effect on the susceptibility toward HPV infection and the persistence of the infection. Genetic predisposition to cervical cancer is related to HLA class II. HLA, B7 and DQB1 are positively associated with cervical neoplasm [9]. However, several other candidate genes in the MHC regions except of the HLA such as TNF [10], LTA [11], TAP and TAP [12], which are part of different pathways, have been suggested to influence cervical cancer. Then, cervical cancer is a complex disease that results from the interaction between gene mutations and the environment [13].

TNF- $\alpha$ is one of the most intensively studied molecule in the field of immunology and cancer [14-15]. TNF- $\alpha$ is an important pleiotropic inflammatory cytokine exerting both homeostatic and pathophysiological function in the periphery and in the central nervous system (CNS), which plays a critical role in the pathogenesis of several autoimmune diseases $[16,17]$. The TNF- $\alpha$ gene is located on the short arm of chromosome 6p21.1-p21.3 and various polymorphisms in this gene have been identified with susceptibility to cancers such as TNF- $\alpha-308 \mathrm{G}>\mathrm{A}$ (rs1800629), TNF- $\alpha-857 \mathrm{~T}>\mathrm{C}$ (rs1799724), TNF-T-1031C (rs1799964) and TNF- $\alpha-238 \mathrm{G}>\mathrm{A}(\mathrm{rs} 361525)$ [18]. In the past decade, the several epidemiologic studies investigated TNF- $\alpha$ polymorphisms on cervical cancer susceptibility. However, the results remain fairly inconsistent and inconclusive. To derive a more precise estimation of the association between TNF- $\alpha$ polymorphisms and cervical cancer risk, we conducted a meta-analysis of all available case-control studies relating the TNF- $\alpha$ rs1800629 and rs 1799724 polymorphisms to the risk of developing cervical cancer.

\section{Materials and Methods}

\section{Search Strategy}

A systematic search of eligible studies on the association between TNF- $\square$ gene polymorphisms and cervical cancer susceptibility was conducted in Medline, ISI Web of Science, Google Scholar, and Embase databases up to the end of April 2017. The following terms were included in the search: "cervical cancer", -308 G>A, s1799724, rs1800629, "'single nucleotide polymorphisms", 'SNPs", "polymorphism", "variant", and "genotype", "SNP", and "allele". The extracted publications were limited to English. References of retrieved articles, review articles and similar meta-analysis were screened for other additional original articles. If there were multiple reports of the same study or overlapping data only the study with the largest sample sizes or the most recent one was include to the meta-analysis.

\section{Inclusion and Exclusion Criteria}

All studies included in this meta-analysis had to meet the following criteria: (1) full-text published studies; (2) studies with case-control or cohort design; (3) a study evaluated the association of TNF- $\alpha$ gene polymorphisms with cervical cancer risk; (4) available genotypes frequencies of TNF- $\alpha$ polymorphisms were provided to estimate the odds ratios (ORs) with $95 \%$ confidence intervals (CIs). The exclusion criteria were as follows: (1) the study was not conducted on cervical cancer; (2) abstracts, case reports, letter to editor, and reviews; (3) studies with only case group (no control group); (4) studies without detail genotype frequencies, which were unable to calculate ORs; and (5) duplicate publications of data from the same study.

\section{Data Extraction}

Two independent authors extracted the information of each eligible study according to the inclusion criteria using a pre-designed form. The following items were extracted from each study: the first author, year of publication, number of cervical cancer patients and controls, genotype and allele frequency, minor allele frequencies (MAFs) in control subjects, and Hardy-Weinberg equilibrium test in control subjects. Any disagreements or conflicting evaluation were resolved by reaching a consensus through discussion or the involvement of a third party.

\section{Statistical Analysis}

The strength of association between the TNF- $\alpha$ polymorphisms and the cervical cancer risk was assessed by ORs with $95 \%$ CIs. The significance of pooled ORs was examined by Z-test. Five different genetic models were used in the current meta-analysis for TNF- $\alpha$ rs 1800629 including the allelic model (A vs. G), the homozygote model (AA vs. GG), the heterozygote model (AG vs. $\mathrm{GG})$, the dominant model (AA+AG vs. GG), and the recessive model (AA vs. AG+GG). The pooled ORs for TNF- $\alpha$ rs1799724 were performed in different genetic comparison models, including the allele model (T vs. C), the homozygote model (TT versus CC), the heterozygote model (TC versus CC), dominant model (TT+TC versus $\mathrm{CC}$ ) and recessive model (TT versus $\mathrm{TC}+\mathrm{CC}$ ). Heterogeneity assumption was checked by a chi-squarebased Q test, and I2 statistics was calculated to quantify the proportion of the total variation across studies due to heterogeneity $[19,20]$. The heterogeneity was considered significant if either the Q statistic had $\mathrm{p}<0.1$ or I $>$ $50 \%$. An I 2 value of $0 \%$ represents no heterogeneity, with values of $25 \%, 50 \%, 75 \%$, or more represent low, moderate, high, and ex $\neg$ treme heterogeneity, 
respectively. A P value greater than 0.10 indicated a lack of heterogeneity among studies, so the fixed effect model (Mantel-Haenszel method) was used to calculate pooled OR. Otherwise, the fixed-effects model (Mantel-Haenszel approach) was used [20-21]. HWEs were calculated with goodness-of-fit tests (i.e., chi-square or Fisher's exact tests). A value of $\mathrm{p}<0.01$ signified a departure from HWE [22]. One-way sensitivity analyses were carried out by consecutively omitting one study at a time to assess the power of the meta-analysis findings. Publication bias was assessed both visually by using a funnel plot and statistically via Begg's funnel plots and Egger's bias test $(p<0.05$ was considered statistically significant), which measures the degree of funnel plot asymmetry [23]. Sensitivity analysis was performed to evaluate the stability of the results by removing the studies. All the statistical analyses were performed by comprehensive meta-analysis (CMA) version 2.0 software (Biostat, USA). All p-values were two-tailed with a significant level at 0.05 .

\section{Results}

Through electronic search, a total of 38 relevant studies concerning TNF- $\alpha$ rs1800629 and rs1799724 polymorphisms and cervical cancer risk were selected following an initial search, which 24 case control studies fit the inclusion criteria. Of the 14 excluded studies, two articles were reviews, seven were redundant studies, four were not involved with TNF- $\alpha$ polymorphism and one study was excluded because did not report allele frequencies for controls used for calculating ORs and $95 \%$ CIs. For the rs 1800629 polymorphism, 4,780 cases and 4,620 controls were available from four studies, whereas for the rs 1799724 polymorphism, 828 cases and 871 controls were available from six studies. Overall, eleven studies used Caucasians, ten used Asians, and three studies used African populations. The countries of eligible studies included Korea (one study), USA (four studies), Zimbabwe (one study), Portugal (one study), South Africa (one study), India (three studies), China (six studies), Sweden (one study), Argentina (two studies), Tunisia (one study), Poland (one study) and Mexico (one study). The results of HWE test for the distribution of the genotype in healthy control populations are shown in Table 1. The genotype distribution in six case-control studies was not in agreement with HWE $(\mathrm{p}<0.005)$.

\section{Quantitative Synthesis}

\section{$T N F-\alpha$ rs 1800629}

Table 2 listed the main results of the meta-analysis of TNF- $\alpha$ rs 1800629 polymorphism and cervical cancer risk. When all the eligible studies were pooled into the meta-analysis of rs1800629 polymorphism, significantly increased risk of cervical cancer was observed in the allelic model (A vs. G: OR $=1.277,95 \% \mathrm{CI}=1.104-1.477, \mathrm{P}$ $=0.001$, Figure 1A), the homozygote model (AA vs. GG: $\mathrm{OR}=1.333,95 \% \mathrm{CI}=1.062-1.674, \mathrm{P}=0.013)$, the heterozygote model (AG vs. GG: $\mathrm{OR}=1.307,95 \% \mathrm{CI}=$
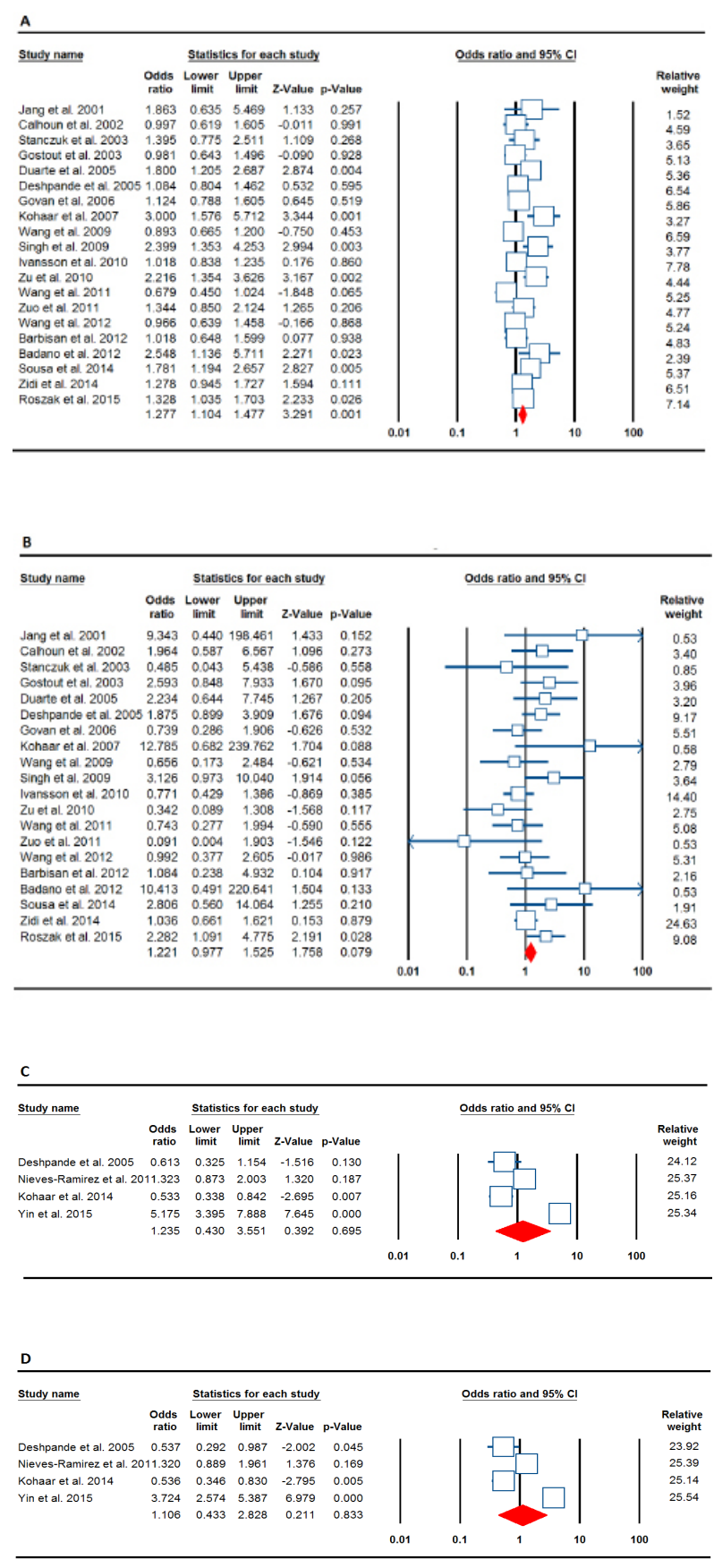

Figure 1. Forest Plots Showed Significant Association between TNF- $\alpha$ rs1800629 and rs1799724 Polymorphisms and Cervical Cancer Risk. A, rs1800629 (allele model, A vs. G); B, rs1800629 (recessive model, AA vs. AG+GG); C, rs1799724 (heterozygote model, TC vs. CC); D, rs1799724 (dominant model, TT+TC vs. CC)

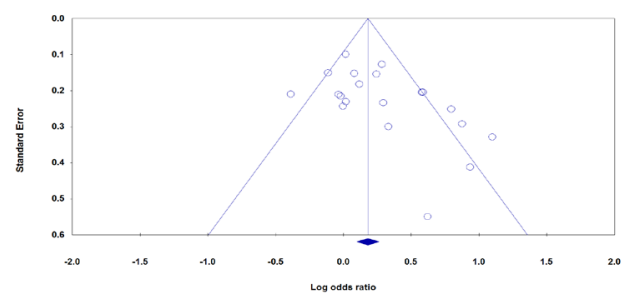

Figure 2. Begg's Funnel Plots of TNF- $\alpha$ rs1800629 Polymorphism and Cervical Cancer Risk under the Allele Model for Publication Bias Test. Each point represents a separate study for the indicated association 
Table 1. Characteristics of Studies Included in TNF $\alpha$ rs1800629 and rs1799724 Polymorphisms and Cervical Cancer

\begin{tabular}{|c|c|c|c|c|c|c|c|c|c|c|c|c|c|c|}
\hline \multirow{3}{*}{$\begin{array}{l}\text { First author } \\
\text { TNF } \alpha \\
\text { rs1800629 }\end{array}$} & \multirow{3}{*}{ Country ethnicity } & \multirow{3}{*}{ Case/Control } & \multicolumn{5}{|c|}{ Cases } & \multicolumn{5}{|c|}{ Controls } & \multirow{3}{*}{ MAFs } & \multirow{3}{*}{ HWE } \\
\hline & & & \multicolumn{3}{|c|}{ Genotype } & \multicolumn{2}{|c|}{ Allele } & \multicolumn{3}{|c|}{ Genotype } & \multicolumn{2}{|c|}{ Allele } & & \\
\hline & & & GG & $\mathrm{AG}$ & AA & G & A & GG & $\mathrm{AG}$ & AA & G & A & & \\
\hline $\begin{array}{l}\text { Jang et al. } \\
2001 \text { [24] }\end{array}$ & Korea (Asian) & $51 / 92$ & 46 & 3 & 2 & 95 & 7 & 85 & 7 & 0 & 177 & 7 & 0.038 & 0.704 \\
\hline $\begin{array}{l}\text { Calhoun et al. } \\
2002 \text { [25] }\end{array}$ & USA(Caucasian) & $127 / 107$ & 91 & 27 & 9 & 209 & 45 & 73 & 30 & 4 & 176 & 38 & 0.177 & 0.678 \\
\hline $\begin{array}{l}\text { Stanczuk et } \\
\text { al. } 2003 \text { [26] }\end{array}$ & Zimbabwe (African) & $103 / 101$ & 74 & 28 & 1 & 176 & 30 & 81 & 18 & 2 & 180 & 22 & 0.108 & 0.41 \\
\hline $\begin{array}{l}\text { Gostout et al. } \\
2003 \text { [12] }\end{array}$ & USA (Caucasian) & $127 / 175$ & 91 & 27 & 9 & 209 & 45 & 117 & 53 & 5 & 287 & 63 & 0.18 & 0.731 \\
\hline $\begin{array}{l}\text { Duarte et al. } \\
2005 \text { [27] }\end{array}$ & Portugal (Caucasian) & $195 / 244$ & 138 & 50 & 7 & 326 & 64 & 200 & 40 & 4 & 440 & 48 & 0.098 & 0.236 \\
\hline $\begin{array}{l}\text { Deshpande et } \\
\text { al. } 2005 \text { [28] }\end{array}$ & USA (Caucasian) & $258 / 411$ & 188 & 54 & 16 & 430 & 86 & 297 & 100 & 14 & 694 & 128 & 0.155 & 0.13 \\
\hline $\begin{array}{l}\text { Govan et al. } \\
2006 \text { [29] }\end{array}$ & South Africa (African) & $244 / 228$ & 174 & 62 & 8 & 410 & 78 & 172 & 46 & 10 & 390 & 66 & 0.144 & 0.005 \\
\hline $\begin{array}{l}\text { Kohaar et al. } \\
2007 \text { [16] }\end{array}$ & India (Asian) & $120 / 165$ & 94 & 22 & 4 & 210 & 30 & 150 & 15 & 0 & 315 & 15 & 0.045 & 0.54 \\
\hline $\begin{array}{l}\text { Wang et al. } \\
2009 \text { [30] }\end{array}$ & China (Asian) & $456 / 800$ & 386 & 67 & 3 & 839 & 73 & 666 & 126 & 8 & 1458 & 142 & 0.088 & 0.457 \\
\hline $\begin{array}{l}\text { Singh et al. } \\
2009 \text { [31] }\end{array}$ & India (Asian) & $150 / 162$ & 122 & 17 & 11 & 261 & 39 & 147 & 11 & 4 & 305 & 19 & 0.058 & $\leq 0.001$ \\
\hline $\begin{array}{l}\text { Ivansson et al. } \\
2010 \text { [32] }\end{array}$ & Sweden (Caucasian) & $1263 / 552$ & 891 & 340 & 32 & 2122 & 404 & 396 & 138 & 18 & 930 & 174 & 0.157 & 0.169 \\
\hline $\begin{array}{l}\text { Zu et al. } 2010 \\
\text { [33] }\end{array}$ & China (Asian) & $83 / 91$ & 30 & 50 & 3 & 110 & 56 & 66 & 16 & 9 & 148 & 34 & 0.186 & $\leq 0.001$ \\
\hline $\begin{array}{l}\text { Wang et al. } \\
2011 \text { [34] }\end{array}$ & China (Asian) & $186 / 200$ & 149 & 30 & 7 & 328 & 44 & 144 & 46 & 10 & 334 & 66 & 0.165 & 0.019 \\
\hline $\begin{array}{l}\text { Zuo et al. } \\
2011 \text { [35] }\end{array}$ & China (Asian) & $239 / 110$ & 158 & 81 & 0 & 397 & 81 & 83 & 25 & 2 & 191 & 29 & 0.131 & 0.941 \\
\hline $\begin{array}{l}\text { Wang et al. } \\
2012 \text { [36] }\end{array}$ & China (Asian) & $285 / 318$ & 247 & 30 & 8 & 524 & 46 & 274 & 35 & 9 & 583 & 53 & 0.083 & $\leq 0.001$ \\
\hline $\begin{array}{l}\text { Barbisan et al. } \\
2012 \text { [37] }\end{array}$ & Argentina (Caucasian) & $122 / 176$ & 87 & 32 & 3 & 206 & 38 & 126 & 46 & 4 & 298 & 54 & 0.153 & 0.483 \\
\hline $\begin{array}{l}\text { Badano et al. } \\
2012 \text { [14] }\end{array}$ & Argentina (Caucasian) & $56 / 113$ & 44 & 10 & 2 & 98 & 14 & 101 & 12 & 0 & 214 & 12 & 0.053 & 0.551 \\
\hline $\begin{array}{l}\text { Sousa et al. } \\
2014 \text { [15] }\end{array}$ & Portugal (Caucasian) & $223 / 205$ & 152 & 65 & 6 & 369 & 77 & 164 & 39 & 2 & 367 & 43 & 0.104 & 0.849 \\
\hline $\begin{array}{l}\text { Zidi et al. } \\
2014 \text { [38] }\end{array}$ & Tunisia (African) & $130 / 260$ & 55 & 33 & 43 & 143 & 117 & 141 & 35 & 84 & 317 & 203 & 0.39 & $\leq 0.001$ \\
\hline $\begin{array}{l}\text { Roszak et al. } \\
2015 \text { [39] }\end{array}$ & Poland (Caucasian) & $362 / 399$ & 217 & 123 & 22 & 557 & 167 & 263 & 125 & 11 & 651 & 147 & 0.184 & 0.397 \\
\hline $\begin{array}{l}\text { TNF } \alpha \\
\text { rs1799724 }\end{array}$ & & $4780 / 4620$ & $\mathrm{CC}$ & $\mathrm{TC}$ & $\mathrm{TT}$ & $\mathrm{C}$ & $\mathrm{T}$ & $\mathrm{CC}$ & $\mathrm{TC}$ & $\mathrm{TT}$ & $\mathrm{C}$ & $\mathrm{T}$ & & \\
\hline $\begin{array}{l}\text { Deshpande et } \\
\text { al. } 2005 \text { [28] }\end{array}$ & USA (Caucasian) & $139 / 115$ & 116 & 22 & 1 & 254 & 24 & 84 & 26 & 5 & 194 & 36 & 0.156 & 0.123 \\
\hline $\begin{array}{l}\text { Nieves- } \\
\text { Ramirez et al. } \\
2011 \text { [40] }\end{array}$ & Mexico [Caucasian) & $191 / 205$ & 93 & 82 & 16 & 268 & 114 & 114 & 76 & 15 & 304 & 106 & 0.258 & 0.636 \\
\hline $\begin{array}{l}\text { Kohaar et al. } \\
2014 \text { [16] }\end{array}$ & India (Asian) & $150 / 200$ & 99 & 44 & 7 & 242 & 58 & 102 & 85 & 13 & 289 & 111 & 0.277 & 0.397 \\
\hline $\begin{array}{l}\text { Yin et al. } \\
2015 \text { [41] }\end{array}$ & China (Asian) & $348 / 351$ & 215 & 87 & 46 & 517 & 179 & 301 & 44 & 6 & 646 & 56 & 0.08 & 0.006 \\
\hline
\end{tabular}

1.064-1.605, $\mathrm{P}=0.011)$, the dominant model $(\mathrm{AA}+\mathrm{AG}$ vs. $\mathrm{GG}: \mathrm{OR}=1.324,95 \% \mathrm{CI}=1.104-1.587, \mathrm{P}=0.002)$, but not under the recessive model (AA vs. AG+GG: OR $=1.221,95 \% \mathrm{CI}=0.977-1.525, \mathrm{P}=0.079$, Figure $1 \mathrm{~B})$. Stratified analysis by ethnicity showed no association between TNF $\alpha$ rs 1800629 polymorphism and cervical cancer risk in Asians under all genetic models. However, significantly increased autism risk was observed in Africans (heterozygote model: $\mathrm{AG}$ vs. $\mathrm{GG}, \mathrm{OR}=1.670$, $95 \% \mathrm{CI}=1.228-2.270, \mathrm{P}=0.001$ and dominant model: $\mathrm{AA}+\mathrm{AG}$ vs. $\mathrm{GG}, \mathrm{OR}=1.453,95 \% \mathrm{CI}=1.111-1.902$, $\mathrm{P}=0.006$ ) and Caucasians (allelic model: A vs. G, OR 
Table 2. The Meta-Analysis of TNF $\alpha$ rs 1800629 Polymorphism and Cervical Cancer Risk

\begin{tabular}{|c|c|c|c|c|c|c|c|c|c|c|c|}
\hline \multirow{2}{*}{ Polymorphism } & \multirow{2}{*}{$\begin{array}{c}\text { Study } \\
\text { Number }\end{array}$} & \multirow{2}{*}{ Genetic Model } & \multirow{2}{*}{$\begin{array}{l}\text { Type of } \\
\text { Model }\end{array}$} & \multicolumn{2}{|c|}{ Heterogeneity } & \multicolumn{4}{|c|}{ Odds Ratio } & \multicolumn{2}{|c|}{ Publication Bias } \\
\hline & & & & $\mathrm{I}^{2}(\%)$ & $\mathrm{P}_{\mathrm{H}}$ & OR & $95 \% \mathrm{CI}$ & $Z_{\text {test }}$ & $\mathrm{P}_{\mathrm{OR}}$ & $\mathrm{P}_{\text {Beggs }}$ & $\mathrm{P}_{\text {Eggers }}$ \\
\hline \multicolumn{12}{|l|}{ Overall } \\
\hline & 20 & A vs. G & Random & 61.94 & $\leq 0.001$ & 1.277 & $1.104-1.477$ & 3.291 & 0.001 & 0.029 & 0.025 \\
\hline & 20 & AA vs. GG & Fixed & 27.43 & 0.125 & 1.333 & $1.062-1.674$ & 2.481 & 0.013 & 0.314 & 0.366 \\
\hline & 20 & AG vs. GG & Random & 70.89 & $\leq 0.001$ & 1.307 & $1.064-1.605$ & 2.552 & 0.011 & 0.183 & 0.141 \\
\hline & 20 & $\mathrm{AA}+\mathrm{AG}$ vs. GG & Random & 67.34 & $\leq 0.001$ & 1.324 & $1.104-1.587$ & 3.03 & 0.002 & 0.097 & 0.056 \\
\hline & 20 & AA vs. $\mathrm{AG}+\mathrm{GG}$ & Fixed & 35.98 & 0.056 & 1.221 & $0.977-1.525$ & 1.758 & 0.079 & 0.537 & 0.336 \\
\hline \multicolumn{12}{|l|}{ Asian } \\
\hline & 8 & A vs. G & Random & 78.48 & $\leq 0.001$ & 1.403 & $0.970-2.029$ & 1.798 & 0.072 & 0.035 & 0.062 \\
\hline & 8 & AA vs. GG & Fixed & 43.54 & 0.088 & 1.089 & $0.670-1.770$ & 0.343 & 0.731 & 1 & 0.54 \\
\hline & 8 & AG vs. GG & Random & 82.21 & $\leq 0.001$ & 1.469 & $0.895-2.411$ & 1.521 & 0.128 & 0.173 & 0.267 \\
\hline & 8 & $\mathrm{AA}+\mathrm{AG}$ vs. GG & Random & 81.63 & $\leq 0.001$ & 1.5 & $0.954-2.359$ & 1.756 & 0.079 & 0.173 & 0.121 \\
\hline & 8 & AA vs. $\mathrm{AG}+\mathrm{GG}$ & Random & 50.71 & 0.048 & 1.04 & $0.487-2.217$ & 0.1 & 0.92 & 0.901 & 0.647 \\
\hline \multicolumn{12}{|l|}{ African } \\
\hline & 3 & A vs. G & Fixed & 0 & 0.786 & 1.234 & $0.996-1.529$ & 1.925 & 0.054 & 1 & 0.739 \\
\hline & 3 & AA vs. GG & Fixed & 0 & 0.537 & 1.156 & $0.757-1.766$ & 0.672 & 0.502 & 1 & 0.289 \\
\hline & 3 & AG vs. GG & Fixed & 24.821 & 0.264 & 1.67 & $1.228-2.270$ & 3.268 & 0.001 & 1 & 0.564 \\
\hline & 3 & $\mathrm{AA}+\mathrm{AG}$ vs. GG & Fixed & 0 & 0.585 & 1.453 & $1.111-1.902$ & 2.725 & 0.006 & 1 & 0.766 \\
\hline & 3 & AA vs. AG+GG & Fixed & 0 & 0.702 & 0.955 & $0.640-1.425$ & -0.225 & 0.822 & 1 & 0.185 \\
\hline \multicolumn{12}{|l|}{ Caucasian } \\
\hline & 9 & A vs. G & Random & 52.45 & 0.032 & 1.242 & $1.043-1.478$ & 2.438 & 0.015 & 0.754 & 0.203 \\
\hline & 9 & AA vs. GG & Fixed & 22.58 & 0.242 & 1.586 & $1.147-2.193$ & 2.791 & 0.005 & 0.175 & 0.072 \\
\hline & 9 & AG vs. GG & Random & 54.87 & 0.023 & 1.123 & $0.905-1.395$ & 1.056 & 0.291 & 0.754 & 0.906 \\
\hline & 9 & $\mathrm{AA}+\mathrm{AG}$ vs. GG & Random & 52.8 & 0.031 & 1.201 & $0.982-1.469$ & 1.787 & 0.074 & 0.916 & 0.501 \\
\hline & 9 & AA vs. $\mathrm{AG}+\mathrm{GG}$ & Fixed & 22.15 & 0.246 & 1.569 & $1.137-2.165$ & 2.744 & 0.006 & 0.348 & 0.079 \\
\hline
\end{tabular}

$=1.242,95 \% \mathrm{CI}=1.043-1.478, \mathrm{P}=0.015 ;$ homozygote model: AA vs. GG, $\mathrm{OR}=1.586,95 \% \mathrm{CI}=1.147-2.193$, $\mathrm{P}=0.005$; recessive model: $\mathrm{AA}$ vs. $\mathrm{AG}+\mathrm{GG}, \mathrm{OR}=1.569$, $95 \% \mathrm{CI}=1.137-2.165, \mathrm{P}=0.006)$.

\section{TNF- $\alpha$ s 1799724}

Table 3 listed the main results of the meta-analysis of TNF- $\alpha$ s 1799724 polymorphism and cervical cancer risk. When all the eligible studies were pooled into the meta-analysis of s1799724 polymorphism, there was no significant association between TNF- $\alpha$ s1799724 and cervical cancer under all five genetic models (allelic model: $\mathrm{T}$ vs. $\mathrm{C}, \mathrm{OR}=1.133,95 \% \mathrm{CI}=0.452-2.838, \mathrm{P}=$ 0.790; homozygote model: $\mathrm{CT}$ vs. $\mathrm{CC}, \mathrm{OR}=0.735,95 \%$ $\mathrm{CI}=0.356-1.518, \mathrm{P}=0.405$; heterozygote model: TT vs. $\mathrm{CC}, \mathrm{OR}=1.235,95 \% \mathrm{CI}=0.430-3.551, \mathrm{P}=0.695$, Figure $1 \mathrm{C}$; dominant model: $\mathrm{TT}+\mathrm{CT}$ vs. $\mathrm{CC}, \mathrm{OR}=1.106,95 \% \mathrm{CI}$ $=0.433-2.828, \mathrm{P}=0.833$, Figure $1 \mathrm{D}$; and recessive model: TT vs. $\mathrm{CT}+\mathrm{CC}, \mathrm{OR}=1.241,95 \% \mathrm{CI}=0.306-5.045$, $\mathrm{P}=0.762$ ). Stratified analysis by ethnicity showed no association between TNF- $\alpha$ s1799724 polymorphism and cervical cancer risk in Caucasian and Asian populations under all genetic models.

Heterogeneity test and sensitivity analysis

There was significant between-study heterogeneity for both TNF- $\alpha-308 \mathrm{G}>$ A and TNF- $\alpha-857 \mathrm{~T}>$ C polymorphisms
(Table 2). We have also performed sensitivity analysis to explore the potential influence of each individual study on the overall results by deleting one single study each time from the pooled analysis. However, no substantial change was observed in the overall studies, indicating that no individual study could affect the pooled OR significantly (data not shown).

\section{Publication bias}

To examine the publication bias of the currently available literature, both Begg's funnel plot and Egger's test were used. The shape of the funnel plots did not reveal any evidence of obvious asymmetry in all comparison models. Moreover, the Egger's test was used to provide statistical evidence for funnel plot symmetry. The results showed evidence of publication bias for TNF- $\alpha-308 \mathrm{G}>\mathrm{A}$ under allele model (PBeggs $=0.029$ and PEggers $=0.025)$, but not for TNF- $\alpha-857 \mathrm{~T}>\mathrm{C}$ (Figure 2).

\section{Discussion}

This systematic review and meta-analysis aimed to explore the association of s1799724 and rs1800629 polymorphisms of TNF- $\alpha$ and cervical cancer risk from 24 case-control studies with 5608 cases and 5491 healthy controls. Of the 24 included studies, only four involved TNF- $\alpha$ s 1799724 with 828 cases and 871 controls. 
Table 3. The Meta-Analysis of TNF $\alpha$ rs1799724 Polymorphism and Cervical Cancer Risk

\begin{tabular}{|c|c|c|c|c|c|c|c|c|c|c|c|}
\hline \multirow{2}{*}{ Polymorphism } & \multirow{2}{*}{$\begin{array}{l}\text { Study } \\
\text { Number }\end{array}$} & \multirow{2}{*}{ Genetic Model } & \multirow{2}{*}{ Type of Model } & \multicolumn{2}{|c|}{ Heterogeneity } & \multicolumn{4}{|c|}{ Odds Ratio } & \multicolumn{2}{|c|}{$\begin{array}{l}\text { Publication } \\
\text { Bias }\end{array}$} \\
\hline & & & & $\mathrm{I}^{2}(\%)$ & $\mathrm{P}_{\mathrm{H}}$ & OR & $95 \% \mathrm{CI}$ & $Z_{\text {test }}$ & $\mathrm{P}_{\mathrm{OR}}$ & $\mathrm{P}_{\text {Beggs }}$ & $\mathrm{P}_{\text {Eggers }}$ \\
\hline \multicolumn{12}{|l|}{ Overall } \\
\hline & 4 & T vs. C & Random & 95.98 & $\leq 0.001$ & 1.133 & $0.452-2.838$ & 0.266 & 0.79 & 0.734 & 0.444 \\
\hline & 4 & TT vs. CC & Fixed & 48.17 & 0.122 & 0.735 & $0.356-1.518$ & -0.832 & 0.405 & 0.308 & 0.319 \\
\hline & 4 & TC vs. CC & Random & 95.05 & $\leq 0.001$ & 1.235 & $0.430-3.551$ & 0.392 & 0.695 & 0.734 & 0.5 \\
\hline & 4 & $\mathrm{TT}+\mathrm{TC}$ vs. $\mathrm{CC}$ & Random & 94.54 & $\leq 0.001$ & 1.106 & $0.433-2.828$ & 0.211 & 0.833 & 0.308 & 0.293 \\
\hline & 4 & TT vs. $\mathrm{TC}+\mathrm{CC}$ & Random & 86.85 & $\leq 0.001$ & 1.241 & $0.306-5.045$ & 0.303 & 0.762 & 0.308 & 0.581 \\
\hline \multicolumn{12}{|l|}{ Asian } \\
\hline & 2 & T vs. C & Random & 98.25 & $\leq 0.001$ & 1.591 & $0.255-9.924$ & 0.497 & 0.619 & NA & NA \\
\hline & 2 & TT vs. CC & Fixed & 69.36 & 0.071 & 0.882 & $0.455-1.709$ & -0.373 & 0.709 & NA & NA \\
\hline & 2 & TC vs. CC & Random & 98.05 & $\leq 0.001$ & 1.664 & $0.180-15.432$ & 0.448 & 0.654 & NA & NA \\
\hline & 2 & $\mathrm{TT}+\mathrm{TC}$ vs. $\mathrm{CC}$ & Random & 97.73 & $\leq 0.001$ & 1.418 & $0.212-9.475$ & 0.361 & 0.718 & NA & NA \\
\hline & 2 & TT vs. $\mathrm{TC}+\mathrm{CC}$ & Random & 93.28 & $\leq 0.001$ & 2.502 & $0.212-29.587$ & 0.728 & 0.467 & NA & NA \\
\hline \multicolumn{12}{|l|}{ Caucasian } \\
\hline & 2 & T vs. C & Random & 86.4 & 0.007 & 0.813 & $0.346-1.909$ & -0.476 & 0.634 & NA & NA \\
\hline & 2 & TT vs. CC & Fixed & 57.36 & 0.126 & 0.714 & $0.346-1.470$ & -0.915 & 0.36 & NA & NA \\
\hline & 2 & TC vs. CC & Random & 74.77 & 0.046 & 0.936 & $0.442-1.981$ & -0.174 & 0.862 & NA & NA \\
\hline & 2 & $\mathrm{TT}+\mathrm{TC}$ vs. $\mathrm{CC}$ & Random & 83.05 & 0.015 & 0.869 & $0.361-2.092$ & -0.314 & 0.753 & NA & NA \\
\hline & 2 & TT vs. $\mathrm{TC}+\mathrm{CC}$ & Fixed & 65.5 & 0.089 & 0.944 & $0.471-1.890$ & -0.164 & 0.87 & NA & NA \\
\hline
\end{tabular}

The present meta-analysis is the most comprehensive synthesis concerning polymorphisms on TNF- $\alpha$ and susceptibility to cervical cancer. According to our results, there was no an overall significant association of s1799724 polymorphism with cervical cancer risk under all genetic models. Furthermore, stratification by ethnicity indicated no association between the $\mathbf{s} 1799724$ and cervical cancer risk. However, the results showed that rs1800629 polymorphism was significantly associated with the increased cervical cancer risk under four genetic models (A vs. G: OR $=1.277,95 \%$ CI: 1.104-1.477, $\mathrm{p}=$ 0.001; AA vs. GG: OR $=1.333,95 \%$ CI: 1.062-1.674, $\mathrm{p}=0.013$; AG vs. GG: $\mathrm{OR}=1.307,95 \% \mathrm{CI}: 1.064-$ $1.605, \mathrm{p}=0.011$; and $\mathrm{AA}+\mathrm{AG}$ vs. GG: $\mathrm{OR}=1.324$, 95\% CI: 1.104-1.587, $\mathrm{p}=0.002$ ). In stratified analysis, there was a significant association between rs1800629 polymorphism and cervical cancer risk in the subgroup of Caucasians and African, but not in Asians. According to the current meta-analysis, there was a variety in terms of s1799724 and rs1800629 polymorphisms of the TNF- $\alpha$ gene distribution in the different ethnicity. Compared to the previously published meta-analyses $[24,25]$ there are more studies included in the current meta-analysis, and the overall sample size is larger; therefore, our findings are more precise and reliable. In addition, the present meta-analysis there is the only study that has assessed both s1799724 and rs1800629 polymorphisms association with cervical cancer simultaneously. Additionally, our results were not consistent with a meta-analysis by Jin et al., 2015 on rs1800629 polymorphism with cervical cancer risk. They included 18 case-control studies with 2,775 cases and 2,759 controls of rs 1800629 . Their results suggested that rs 1800629 polymorphism was associated with increased cervical cancer risk in both Asian and
Caucasian populations. Additionally, Jin et al., 2015 not performed further subgroup by ethnicity in the African populations to detect significant difference [25]. In the present systematic review and meta-analysis, by including 20 case-control studies with 4,780 cases and 4,620 controls for quantitative synthesis, we found that the rs 1800629 polymorphism was associated with cervical cancer risk in Caucasians and Africans, but not Asian populations.

Heterogeneity is a potential problem that may affect the accuracy of the meta-analyses results [26, 27]. Heterogeneity may be due to many factors, such as differences in the small sample size, diversity in design, inclusion criteria, diverse genotyping method, characteristics of controls, and a mixed population from different ethnicity $[28,29]$. In present meta-analysis a significant heterogeneity was found for rs 1800629 (under allele, heterozygote and dominant models) and rs 1799724 (under allele, heterozygote, dominant and recessive models) polymorphisms in the overall population. Thus, we conducted subgroup analysis by ethnicity and found a statistically significant level of heterogeneity for rs1800629 in the Asian and Caucasian populations heterogeneity is still exist in, but not in Africans. A similar result was found for rs 1799724 . Therefore, both polymorphisms were the sources of the heterogeneity. It was suggested that different allelic frequencies in different ethnic groups may account for these discrepancies [30]. Moreover, in the sensitivity analysis, we have not found significant after omitting each study at a time, indicating the relative stability and credibility of the results of our meta-analysis.

This meta-analysis had significantly higher statistical power than the previous meta-analyses that analyzed the association between the TNF- $\alpha$ polymorphism and 
cervical cancer risk, since the cancer patients involved in our meta-analysis were higher as many as the previous one. However, some limitation should be considered in this meta-analysis. First, the number of available studies for TNF- $\alpha$ rs 1799724 polymorphism was limited to the four case-control studies, and, due to the limited sample size, the pooled results were less accurate and more studies with large sample size and high quality are needed for further analysis. Second, in the present meta-analysis we have included only published studies; therefore, publication bias might have occurred and the present meta-analysis results may have a substantial risk of being affected by bias. Third, the heterogeneity is difficult to exclude, in that it is influenced by complicated factors, such as age, sex, genetic diversities, different lifestyle, and clinical characteristics. In this study, a significant between study heterogeneity was found in most of the meta-analyses for both polymorphisms. Reduced heterogeneity was observed in some ethnicity after subgroup analyses, especially in African populations. However, due to the complexity of cervical cancer and potential confounding factors such as age, infection with HPV, lifestyle, difference in clinical and/or environmental factors might have contributed to the heterogeneity among individual studies. Finally, due to limited individual data for the adjustments of major confounders, we did not conduct a more precise analysis on other covariates such as age, lifestyle, HPV infection, and environmental factors.

In summary, this meta-analysis of 24 case-control studies suggested that the rs1800629 polymorphism of the TNF- $\alpha$ gene was significantly associated with cervical cancer risk, but not s1799724. Moreover, compared with Asians, African and Caucasian female with A allele of the TNF $\alpha$ rs 1800629 had been found to have a greater susceptibility for the development of cervical cancer. Additionally, due to the limited number of studies and sample size included for TNF- $\alpha$ rs 1799724 polymorphism, well-designed studies with large sample size and more ethnic groups are required to further verify and confirm current meta-analysis results.

\section{References}

1. Jemal A, Bray F, Center MM, Ferlay J, Ward E, Forman D. Global cancer statistics. CA: a cancer journal for clinicians. 2011;61(2):69-90.

2. Yazdi MF, Rafieian S, Gholi-Nataj M, Sheikhha MH, Nazari T, Neamatzadeh H. CYP2D6 Genotype and Risk of Recurrence in Tamoxifen Treated Breast Cancer Patients. Asian Pacific journal of cancer prevention : APJCP. 2015;16(15):6783-7.

3. Arbyn M, Castellsague X, de Sanjose S, Bruni L, Saraiya M, Bray F, et al. Worldwide burden of cervical cancer in 2008. Annals of oncology : official journal of the European Society for Medical Oncology. 2011;22(12):2675-86.

4. Sherris J, Herdman C, Elias C. Cervical cancer in the developing world. The Western journal of medicine. 2001;175(4):231-3.

5. Monk BJ, Herzog TJ. The new era of cervical cancer prevention: HPV vaccination. Gynecologic oncology. 2008;109(2 Suppl):S1-3.

6. Catarino R, Petignat P, Dongui G, Vassilakos P. Cervical cancer screening in developing countries at a crossroad: Emerging technologies and policy choices. World journal of clinical oncology. 2015;6(6):281-90.

7. Hemminki K, Dong C, Vaittinen P. Familial risks in cervical cancer: is there a hereditary component? International journal of cancer. 1999;82(6):775-81.

8. Magnusson PK, Sparen P, Gyllensten UB. Genetic link to cervical tumours. Nature. 1999;400(6739):29-30.

9. Engelmark M, Beskow A, Magnusson J, Erlich H, Gyllensten U. Affected sib-pair analysis of the contribution of HLA class I and class II loci to development of cervical cancer. Human molecular genetics. 2004;13(17):1951-8.

10. Jang WH, Yang YI, Yea SS, Lee YJ, Chun JH, Kim HI, et al. The -238 tumor necrosis factor-alpha promoter polymorphism is associated with decreased susceptibility to cancers. Cancer letters. 2001;166(1):41-6.

11. Niwa Y, Hirose K, Matsuo K, Tajima K, Ikoma Y, Nakanishi $\mathrm{T}$, et al. Lymphotoxin-alpha polymorphism and the risk of cervical cancer in Japanese subjects. Cancer letters. 2005;218(1):63-8

12. Gostout BS, Poland GA, Calhoun ES, Sohni YR, Giuntoli RL, 2nd, McGovern RM, et al. TAP1, TAP2, and HLA-DR2 alleles are predictors of cervical cancer risk. Gynecologic oncology. 2003;88(3):326-32.

13. Martínez-Nava G F-NJ, Madrid-Marina V, Madrid-Marina V, Torres-Poveda K. Cervical Cancer Genetic Susceptibility: A Systematic Review and Meta-Analyses of Recent Evidence. PLOS ONE. e0157344. 2016;

14. Badano I, Stietz SM, Schurr TG, Picconi AM, Fekete D, Quintero IM, et al. Analysis of TNFalpha promoter SNPs and the risk of cervical cancer in urban populations of Posadas (Misiones, Argentina). Journal of clinical virology : the official publication of the Pan American Society for Clinical Virology. 2012;53(1):54-9.

15. Sousa H, Oliveira S, Santos AM, Catarino R, Moutinho J, Medeiros R. Tumour necrosis factor alpha $308 \mathrm{G} / \mathrm{A}$ is a risk marker for the progression from high-grade lesions to invasive cervical cancer. Tumour biology : the journal of the International Society for Oncodevelopmental Biology and Medicine. 2014;35(3):2561-4.

16. Kohaar I, Thakur N, Salhan S, Batra S, Singh V, Sharma A, et al. TNFalpha-308G/A polymorphism as a risk factor for HPV associated cervical cancer in Indian population. Cellular oncology : the official journal of the International Society for Cellular Oncology. 2007;29(3):249-56.

17. Probert L. TNF and its receptors in the CNS: The essential, the desirable and the deleterious effects. Neuroscience. 2015;302:2-22.

18. Qidwai T, Khan F. Tumour necrosis factor gene polymorphism and disease prevalence. Scandinavian journal of immunology. 2011;74(6):522-47.

19. Higgins JP, Thompson SG. Quantifying heterogeneity in a meta-analysis. Statistics in medicine. 2002;21(11):1539-58.

20. DerSimonian R, Laird N. Meta-analysis in clinical trials. Controlled clinical trials. 1986;7(3):177-88.

21. Mantel N, Haenszel W. Statistical aspects of the analysis of data from retrospective studies of disease. Journal of the National Cancer Institute. 1959;22(4):719-48.

22. Khoram-Abadi KM, Forat-Yazdi M, Kheirandish S, Saeidi N, Zarezade Z, Mehrabi N, et al. DNMT3B -149 C $>$ T and $-579 \mathrm{G}>\mathrm{T}$ Polymorphisms and Risk of Gastric and Colorectal Cancer: a Meta-analysis. Asian Pacific journal of cancer prevention : APJCP. 2016;17(6):3015-20.

23. Egger M, Davey Smith G, Schneider M, Minder C. Bias in meta-analysis detected by a simple, graphical test. BMJ (Clinical research ed). 1997;315(7109):629-34. 
24. Cai J, Yang MY, Hou N, Li X. Association of tumor necrosis factor-alpha 308G/A polymorphism with urogenital cancer risk: a systematic review and meta-analysis. Genetics and molecular research : GMR. 2015;14(4):16102-12.

25. Y. J. Association of Single Nucleotide Polymorphisms in Tumor Necrosis Factor-Alpha with Cervical Cancer Susceptibility. 2015 77-84. p.

26. Kamali M, Kargar S, Heiranizadeh N, Zare M, Kargar S, Zare Shehneh M, et al. Lack of any Association between the Hogg1 Ser326Cys Polymorphism and Breast Cancer Risk: a Systematic Review And Meta-Analysis Of 18 Studies. Asian Pacific journal of cancer prevention : APJCP. 2017;18(1):245-51.

27. Sobhan MR, Forat Yazdi M, Mazaheri M, Zare Shehneh M, Neamatzadeh H. Association between the DNA Repair Gene XRCC3 rs861539 Polymorphism and Risk of Osteosarcoma: a Systematic Review and Meta-Analysis. Asian Pacific journal of cancer prevention : APJCP. 2017;18(2):549-55.

28. Qi X, Wan Y, Zhan Q, Yang S, Wang Y, Cai X. Effect of CDKN2A/B rs4977756 polymorphism on glioma risk: a meta-analysis of 16 studies including 24077 participants. Mammalian genome : official journal of the International Mammalian Genome Society. 2016;27(1-2):1-7.

29. Mehdinejad M, Sobhan MR, Mazaheri M, Zare Shehneh M, Neamatzadeh H, Kalantar SM. Genetic Association between ERCC2, NBN, RAD51 Gene Variants and Osteosarcoma Risk: a Systematic Review and Meta-Analysis. Asian Pacific journal of cancer prevention : APJCP. 2017;18(5):1315-21.

30. Yang H, Gao Y, Feng T, Jin TB, Kang LL, Chen C. Metaanalysis of the rs4779584 polymorphism and colorectal cancer risk. PLoS One. 2014;9(2):e89736.

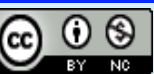

This work is licensed under a Creative Commons AttributionNon Commercial 4.0 International License. 